\title{
Determinants of plasma 25-hydroxyvitamin $D$ and development of prediction models in three US cohorts
}

\author{
Kimberly A. Bertrand ${ }^{1,2 *}$, Edward Giovannucci ${ }^{1,2,3}$, Yan Liu $^{3}$, Susan Malspeis ${ }^{1}$, A. Heather Eliassen ${ }^{2}$, \\ Kana $\mathrm{Wu}^{3}$, Michelle D. Holmes ${ }^{1,2}$, Francine Laden ${ }^{1,2,4}$ and Diane Feskanich ${ }^{2}$ \\ ${ }^{1}$ Department of Epidemiology, Harvard School of Public Health, 677 Huntington Avenue, Boston, MA O2115, USA \\ ${ }^{2}$ Channing Laboratory, Department of Medicine, Brigham and Women's Hospital, and Harvard Medical School, Boston, \\ MA, USA \\ ${ }^{3}$ Department of Nutrition, Harvard School of Public Health, Boston, MA, USA \\ ${ }^{4}$ Department of Environmental Health, Harvard School of Public Health, Boston, MA, USA
}

(Submitted 13 June 2011 - Final revision received 9 November 2011 - Accepted 13 December 2011 - First published online 23 January 2012)

\section{Abstract}

Epidemiological and other evidence suggests that vitamin D may be protective against several chronic diseases. Assessing vitamin D status in epidemiological studies, however, is challenging given finite resources and limitations of commonly used approaches. Using multivariable linear regression, we derived predicted 25-hydroxyvitamin D (25(OH)D) scores based on known determinants of circulating $25(\mathrm{OH}) \mathrm{D}$, including age, race, UV-B radiation flux at residence, dietary and supplementary vitamin D intakes, BMI, physical activity, alcohol intake, post-menopausal hormone use (women only) and season of blood draw, in three nationwide cohorts: the Nurses' Health Study, Nurses' Health Study II and the Health Professionals Follow-up Study. The model $r^{2}$ for each cohort ranged from $0 \cdot 25$ to $0 \cdot 33$. We validated the prediction models in independent samples of participants from these studies. Mean measured 25(OH)D levels rose with increasing decile of predicted 25(OH)D score, such that the differences in mean measured 25(OH)D between the extreme deciles of predicted $25(\mathrm{OH}) \mathrm{D}$ were in the range $8.7-12.3 \mathrm{ng} / \mathrm{ml}$. Substituting predicted $25(\mathrm{OH}) \mathrm{D}$ scores for measured $25(\mathrm{OH}) \mathrm{D}$ in a previously published case-control analysis of colorectal cancer yielded similar effect estimates with OR of approximately $0.8 \mathrm{for}$ a $10 \mathrm{ng} / \mathrm{ml}$ difference in either plasma or predicted $25(\mathrm{OH}) \mathrm{D}$. We conclude that these data provide reasonable evidence that a predicted $25(\mathrm{OH}) \mathrm{D}$ score is an acceptable marker for ranking individuals by long-term vitamin D status and may be particularly useful in research settings where biomarkers are not available for the majority of a study population.

Key words: 25-Hydroxyvitamin D: Epidemiology: Predictors: Vitamin D

Epidemiological evidence suggests that vitamin D may protect against colorectal, prostate, breast and oropharyngeal cancers $^{(1-6)}$ and other chronic diseases such as CVD $^{(6-9)}$, diabetes $^{(6,9-11)}$ and hip fractures ${ }^{(12-14)}$. Plasma 25-hydroxyvitamin D $(25(\mathrm{OH}) \mathrm{D})$, the primary circulating form of vitamin $\mathrm{D}^{(6,15)}$, is an accepted biomarker for measuring vitamin $\mathrm{D}$ status in clinical settings ${ }^{(16)}$; however, it is strongly dependent on season of blood draw. Although 25(OH)D is fairly reproducible over $2-3$ years $^{(17,18)}$, one measurement is weaker in characterising longer-term exposure ${ }^{(19,20)}$. Furthermore, measuring $25(\mathrm{OH}) \mathrm{D}$ requires the availability of blood samples and monetary resources for laboratory assays, thereby limiting the feasibility of this approach for many large-scale epidemiological studies.
Individual determinants of vitamin D status, such as latitude and regional estimates of solar radiation ${ }^{(21-23)}$ or dietary assessment $^{(24-26)}$, have been used as surrogates of vitamin D exposure, but each alone contributes a small proportion of $25(\mathrm{OH}) \mathrm{D}$. An alternative approach to assess vitamin D status is to combine known determinants of circulating $25(\mathrm{OH}) \mathrm{D}$ to derive a predicted score from questionnaire data using measurements of plasma 25(OH)D available for a subset of the study population; reported $r^{2}$ from such predictive models have ranged from $0 \cdot 21$ to $0 \cdot 42^{(18,27-29)}$. Although the $r^{2}$ between predicted and measured 25(OH)D has been used to assess the 'validity' of the predicted $25(\mathrm{OH}) \mathrm{D}$ approach, the $r^{2}$ in this context has limitations, given that a single measure is not a true 'gold standard' of long-term average 25(OH)D concentration. Such an 'alloyed' or imperfect

Abbreviations: 25(OH)D, 25-hydroxyvitamin D; HPFS, Health Professionals Follow-up Study; ICC, intraclass correlation coefficients; NHS, Nurses' Health Study; NHSII, Nurses' Health Study II; Q, quintile.

*Corresponding author: Dr K. A. Bertrand, fax +1 617525 2008, email kbertran@hsph.harvard.edu 
'gold standard' will underestimate true 'validity'(30-32). More importantly, a comparison of high $v$. low circulating $25(\mathrm{OH}) \mathrm{D}$ level in the population, which may be estimated by high and low predicted $25(\mathrm{OH}) \mathrm{D}$, may be the more relevant factor for testing exposure-disease hypotheses in epidemiological studies.

In the present paper, we describe the development and validation of regression models to predict $25(\mathrm{OH}) \mathrm{D}$ based on the determinants of vitamin D status in three cohorts: the Nurses' Health Study (NHS), the Nurses' Health Study II (NHSII) and the Health Professionals Follow-up Study (HPFS). Predicted $25(\mathrm{OH}) \mathrm{D}$ scores have been used in several analyses within these cohorts ${ }^{(8,18,33,34)}$ but, with the exception of $\operatorname{HPFS}^{(18)}$, no formal validation has been conducted previously and the specific prediction models varied with each analysis. We also evaluated the reproducibility of plasma $25(\mathrm{OH}) \mathrm{D}$ over 10-11 years in NHS participants.

\section{Methods}

\section{Study population}

Participants were selected from three US prospective cohort studies. The NHS was established in 1976, when 121700 female nurses aged 30-55 years completed a self-administered questionnaire on risk factors for cancer and other diseases. During 1989 and 1990, a total of 32826 participants provided blood samples for analysis. The NHSII began in 1989 when 116671 female nurses aged 25-42 years completed and returned a baseline questionnaire. Between 1996 and 1999, a total of 29611 participants (aged 32-54 years) provided blood samples. The HPFS comprises 51529 male dentists, optometrists, osteopaths, podiatrists, pharmacists and veterinarians aged 40-75 years at baseline in 1986. Blood samples were provided by 18225 of these men during 1993 and 1994. Blood samples have been stored in liquid $\mathrm{N}_{2}$ freezers $\left(\leq-130^{\circ} \mathrm{C}\right)$ since collection. For all three cohorts, biennial questionnaires are sent to participants to update information on risk factors and to identify newly diagnosed diseases. Diet is assessed by a validated semiquantitative FFQ approximately every 4 years ${ }^{(35-38)}$

Plasma 25(OH)D measurements were available from men and women who served as controls in previous nested case-control studies of chronic diseases. None of the participants had a history of cancer at the time of blood draw. For each cohort, we selected two independent samples: a 'training' sample was used to develop the $25(\mathrm{OH}) \mathrm{D}$ prediction model and a 'test' sample served as a validation data set. Training samples comprised controls from all completed and on-going nested case-control studies with 25(OH)D assay results when analyses began. Test samples were drawn from more recently established nested case-control studies as this project unfolded and additional plasma $25(\mathrm{OH}) \mathrm{D}$ assay results became available. Before exclusions for missing data, the training sets consisted of 2246 women in the NHS, 1646 women in the NHSII and 1255 men in the HPFS. An additional 818 women in the NHS, 479 women in the NHSII and 841 men in the HPFS were available for the test sets.
In 2000 and 2001, all women in the NHS who gave blood in 1989-1990 and were alive were invited to provide a second blood sample. Of the 18473 women who participated in the second blood collection, 443 women with no history of cancer had measured $25(\mathrm{OH}) \mathrm{D}$ available at both time points. These samples were used to assess within-person variability of plasma $25(\mathrm{OH}) \mathrm{D}$ concentrations over 10-11 years.

This study was approved by the institutional review boards of the Harvard School of Public Health and Brigham and Women's Hospital. All participants gave written informed consent at enrolment.

\section{Laboratory analyses}

Plasma 25(OH)D levels were determined by RIA or chemiluminescence immunoassay, as previously described ${ }^{(39-41)}$, between 1993 and 2010. The time between blood collection and $25(\mathrm{OH}) \mathrm{D}$ assay ranged from 3 to 20 years, with the majority of samples assayed within 14 years of blood collection. The stability of $25(\mathrm{OH}) \mathrm{D}$ in frozen plasma has been previously demonstrated, even for samples stored $>10$ years $^{(42)}$. Intra-assay CV from blinded, replicate, quality-control samples were $<15 \%$ for twenty-three out of twenty-six laboratory batches; the highest CV was $17 \cdot 6 \%$. Mean $25(\mathrm{OH}) \mathrm{D}$ concentrations in training samples were: 28.5 (SD 10.9) $\mathrm{ng} / \mathrm{ml}$ (NHS, $n$ 2079); $26 \cdot 3$ (SD 9.8) $\mathrm{ng} / \mathrm{ml}$ (NHSII, $n$ 1497) and 25.9 (sD $10 \cdot 0) \mathrm{ng} / \mathrm{ml}$ (HPFS, $n$ 911).

\section{Statistical analyses}

Using the training sample for each cohort, we fit a linear regression model to predict measured plasma 25(OH)D (continuous $\mathrm{ng} / \mathrm{ml}$ ) based on known or suspected determinants $^{(18)}$. Age (years), season of blood draw and laboratory batch were included as independent variables in all models to account for known extraneous variation. Other candidate predictor variables were energy-adjusted ${ }^{(43)}$ vitamin D intake from food, vitamin $\mathrm{D}$ intake from supplements, average annual UV-B flux - a composite measure of mean UV-B radiation level reaching the earth's surface that takes into account factors such as latitude, altitude and cloud cover - based on state of residence ${ }^{(44)}$, race/ethnicity, BMI, leisure-time physical activity level, alcohol intake, geographic region of residence (North, South, Midwest, West), smoking history, hair colour, susceptibility to burn, ability to tan and number of lifetime sunburns. Menopausal status, post-menopausal hormone use and age at first birth were also considered for women in the NHS and NHSII. Data were obtained from questionnaires completed closest to blood draw date. Questionnaires were completed within \pm 2 years of blood draw for $\geq 97 \%$ of each sample; the median time period was 5 months before blood draw for the NHS, 3 months after blood draw for the NHSII, and 2 months before blood draw for the HPFS.

For each cohort, we first fit a multivariable linear regression model with all candidate predictors with $P<0.05$ in univariate analyses adjusted for laboratory batch and age. Then, we eliminated non-significant $(P \geq 0.05)$ variables from the model, one at a time, based on the largest $P$ value. The final 
multivariable prediction model includes all statistically significant predictors, plus age, season of blood draw and laboratory batch. The HPFS model is a refinement of the one previously published $^{(18)}$. The general form of the prediction model is: $25(\mathrm{OH}) \mathrm{D}=\beta_{0}+\beta^{\prime} X^{\prime}$, where $\beta_{0}$ represents the intercept and $\beta^{\prime}$ represents the vector of coefficients associated with the vector of predictors, $X^{\prime}$ (see Table 1 ).

Regarding the final sets of predictors, we aimed for consistency between cohorts, while allowing for flexibility in cohort-specific models. Factors statistically significant for one cohort were considered for inclusion in models for the other cohorts regardless of statistical significance, given sufficient biological plausibility (e.g. UV-B flux). We excluded individuals with missing values for predictors except post-menopausal hormone use in the NHSII for which a missing category was created. The final prediction models were fit to 2079 women aged 42-69 years in the NHS, 1497 women aged 32-52 years in the NHSII and 911 men aged 46-81 years in the HPFS.

Based on the regression coefficients for each variable in the prediction model, we calculated a predicted $25(\mathrm{OH}) \mathrm{D}$ score for each individual in the test samples using personal data for covariates. Age, season of blood draw and laboratory batch were not used in the derivation of predicted $25(\mathrm{OH}) \mathrm{D}$ scores. Age is not used in the derivation of predicted $25(\mathrm{OH}) \mathrm{D}$ because it is a strong risk factor for many chronic diseases. By excluding age from the derived score, the ability to control finely for potential confounding by age in epidemiological investigations is retained. Predicted 25(OH)D scores were not calculated if predictor data were missing on the questionnaire closest to blood draw or the previous questionnaire (NHS, $n$ 39; NHSII, $n$ 34; HPFS, $n$ 5). For the test samples, there were 779 women in the NHS, 445 women in the NHSII and 836 men in the HPFS with available 25(OH)D measurements and predicted $25(\mathrm{OH}) \mathrm{D}$ scores.

For validation, we compared predicted $25(\mathrm{OH}) \mathrm{D}$ and actual plasma $25(\mathrm{OH}) \mathrm{D}$ measurements in test samples. Laboratory batch-adjusted Spearman correlation coefficients were calculated to assess agreement between predicted score and actual 25(OH)D levels. We examined actual plasma 25(OH)D measurements according to decile of predicted $25(\mathrm{OH}) \mathrm{D}$ score ${ }^{(18,28)}$ and cross-classified individuals by quintile of both predicted and actual $25(\mathrm{OH}) \mathrm{D}$. Using previously published data from a nested case-control study that examined the association between plasma $25(\mathrm{OH}) \mathrm{D}$ and colorectal cancer in the NHS and HPFS ${ }^{(4)}$, we calculated OR for colorectal cancer for a $10 \mathrm{ng} / \mathrm{ml}$ difference in measured 25(OH)D and then compared the results to analyses that used the predicted $25(\mathrm{OH}) \mathrm{D}$ score. In these analyses, we derived separate predicted scores at each questionnaire year based on current predictor data and calculated the average predicted 25(OH)D from 1986 - the year predicted scores were first derived - to date of diagnosis (or matched date for controls) as the main exposure variable. For both measured and predicted $25(\mathrm{OH}) \mathrm{D}$, pooled estimates were calculated for the NHS and HPFS using a meta-analysis approach described by DerSimonian \& Laird ${ }^{(45)}$.

Finally, we evaluated the reproducibility of $25(\mathrm{OH}) \mathrm{D}$ measurements over 10-11 years among 443 women in the NHS with two blood measures, using a statistical approach previously described ${ }^{(17)}$. We calculated intraclass correlation coefficients (ICC) by dividing the between-person variance by the sum of the within- and between-person variances; a 95\% CI also was calculated. Using a mixed model, we adjusted for age (continuous) by including it as a fixed effect. ICC measures the fraction of total variation that is due to between-person variability. A high value for the ICC reflects a low within-person variation.

Among NHS participants with two 25(OH)D measurements 10-11 years apart, we compared average plasma $25(\mathrm{OH}) \mathrm{D}$ concentration to average predicted $25(\mathrm{OH}) \mathrm{D}$ score over the same time period. We calculated Spearman correlation coefficients based on the residuals of plasma 25(OH)D measurements in each time period from a linear regression model to factor out effects of age and season of blood draw. Because random within-person error can attenuate correlations, we used data from the reproducibility sample to correct for these effects ${ }^{(46,47)}$.

All statistical tests were two-sided and analyses were performed using SAS version 9 for UNIX (SAS Institute, Inc.).

\section{Results}

Using multivariable linear regression in the training set within each cohort, we identified the following independent predictors of age-adjusted plasma $25(\mathrm{OH}) \mathrm{D}$ levels: race, UV-B flux (NHS and HPFS only), dietary vitamin D intake, supplementary vitamin D intake, BMI, physical activity, alcohol intake (NHS and NHSII only), post-menopausal hormone use (NHS only) and season of blood draw (Table 1). Overall, the predictive models explained 25\% (NHSII), 28\% (HPFS) and 33\% (NHS) of the total variability in plasma $25(\mathrm{OH}) \mathrm{D}$ concentration. The strongest predictors of circulating $25(\mathrm{OH}) \mathrm{D}$ generally were race (a proxy for skin pigmentation) and BMI, followed by physical activity, dietary and supplementary vitamin D intake and UV-B flux (NHS and HPFS only). Season also was an important predictor of $25(\mathrm{OH}) \mathrm{D}$, but is not used in the calculation of predicted $25(\mathrm{OH}) \mathrm{D}$ score because it reflects time of blood draw and is not a factor in determining longterm average between-person variation in $25(\mathrm{OH}) \mathrm{D}$. Age was not a significant independent predictor of $25(\mathrm{OH}) \mathrm{D}$ in the NHS or HPFS, but a modest inverse association was observed in the NHSII.

Using the regression coefficients estimated in each training set, we calculated predicted $25(\mathrm{OH}) \mathrm{D}$ scores for participants in the corresponding test samples. The batch-adjusted Spearman correlation coefficients between predicted score and actual $25(\mathrm{OH}) \mathrm{D}$ level were $0 \cdot 23(95 \%$ CI $0 \cdot 16,0 \cdot 29)$ for the NHS, $0.40(95 \%$ CI $0.32,0.47)$ for the NHSII and $0.24(95 \%$ CI $0 \cdot 18,0 \cdot 30$ ) for the HPFS (all $P$ values $<0 \cdot 0001$ ). After further adjusting for age and season of blood draw, correlations were $0 \cdot 23$ (95\% CI 0.16, 0.29; NHS), $0 \cdot 42$ (95\% CI 0.34, 0.49; NHSII) and $0 \cdot 30$ (95\% CI: $0 \cdot 21,0 \cdot 37$; HPFS). In all cohorts, actual plasma $25(\mathrm{OH}) \mathrm{D}$ levels generally rose with increasing decile of predicted $25(\mathrm{OH}) \mathrm{D}$ score (Fig. 1). The differences in mean actual 25(OH)D level between extreme deciles of predicted $25(\mathrm{OH}) \mathrm{D}$ score were $8.7 \mathrm{ng} / \mathrm{ml}(95 \% \mathrm{CI} 5.4,11.9)$ for 
Table 1. Predictors of plasma 25-hydroxyvitamin D $(25(\mathrm{OH}) \mathrm{D})$ level from multiple linear regression models in the Nurses' Health Studies (NHS and NHSII) and the Health Professionals Follow-up Study (HPFS)*

\begin{tabular}{|c|c|c|c|c|c|c|}
\hline \multirow[b]{2}{*}{ Predictor } & \multicolumn{2}{|c|}{$\begin{array}{c}\text { NHS } \\
\left(n \text { 2079; model } r^{2} 0.33\right)\end{array}$} & \multicolumn{2}{|c|}{$\begin{array}{c}\text { NHSII } \\
\left.\text { ( } n \text { 1497; model } r^{2} 0.25\right)\end{array}$} & \multicolumn{2}{|c|}{$\begin{array}{c}\text { HPFS } \\
\left(n 911 ; \text { model } r^{2} 0.28\right)\end{array}$} \\
\hline & $\begin{array}{l}\text { Difference in } 25(\mathrm{OH}) \mathrm{D} \\
(\mathrm{ng} / \mathrm{ml} ; \beta)\end{array}$ & $P$ & $\begin{array}{l}\text { Difference in } 25(\mathrm{OH}) \mathrm{D} \\
(\mathrm{ng} / \mathrm{ml} ; \beta)\end{array}$ & $P$ & $\begin{array}{l}\text { Difference in } 25(\mathrm{OH}) \mathrm{D} \\
(\mathrm{ng} / \mathrm{ml} ; \beta)\end{array}$ & $P$ \\
\hline Intercept & $22 \cdot 69$ & & 35.78 & & 31.94 & \\
\hline Age (years) $\dagger$ & 0.07 & 0.07 & -0.23 & $<0.0001$ & -0.04 & 0.24 \\
\hline Race & & $<0.001$ & & $<0.001$ & & 0.03 \\
\hline White & 0 (referent) & & 0 (referent) & & 0 (referent) & \\
\hline Black & -11.30 & & -6.42 & & -4.89 & \\
\hline Asian & - & & -5.55 & & -4.67 & \\
\hline Hispanic & - & & -6.83 & & - & \\
\hline Other & -1.63 & & 1.98 & & -1.48 & \\
\hline UV-B flux category $\ddagger$ & & $<0.0001$ & & 0.67 & & 0.002 \\
\hline 1 (high) & 0 (referent) & & 0 (referent) & & 0 (referent) & \\
\hline 2 & -2.69 & & -0.16 & & -1.89 & \\
\hline 3 & -1.29 & & -0.66 & & -2.54 & \\
\hline 4 & - & & -0.60 & & -2.66 & \\
\hline 5 (low) & - & & - & & -3.97 & \\
\hline Dietary vitamin $D(\mu \mathrm{g} / \mathrm{d})$ & & $<0.0001$ & & 0.003 & & 0.001 \\
\hline$<2.5$ & 0 (referent) & & 0 (referent) & & 0 (referent) & \\
\hline $2 \cdot 5-<5$ & 0.92 & & 1.56 & & -0.32 & \\
\hline $5-<7.5$ & $2 \cdot 19$ & & 1.87 & & $2 \cdot 37$ & \\
\hline $7.5-<10$ & 3.43 & & 3.55 & & 1.93 & \\
\hline$\geq 10$ & 3.33 & & 2.49 & & $3 \cdot 10$ & \\
\hline Supplementary vitamin $D(\mu \mathrm{g} / \mathrm{d})$ & & $<0.0001$ & & $<0.001$ & & $<0.001$ \\
\hline 0 & 0 (referent) & & 0 (referent) & & 0 (referent) & \\
\hline $0.025-<5$ & 2.85 & & 0.76 & & 2.51 & \\
\hline $5-<10$ & 1.57 & & 2.05 & & 0 & \\
\hline$\geq 10$ & $3 \cdot 15$ & & $2 \cdot 70$ & & 2.54 & \\
\hline $\mathrm{BMI}\left(\mathrm{kg} / \mathrm{m}^{2}\right)$ & 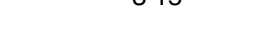 & $<0.0001$ & $2 \pi 0$ & $<0.0001$ & & $<0.001$ \\
\hline$<19$ & - & & $2 \cdot 22$ & & - & \\
\hline$<22$ (19-21.9 in NHSII) & 0 (referent) & & 0 (referent) & & 0 (referent) & \\
\hline $22-24.9$ & -0.57 & & -0.38 & & -0.39 & \\
\hline $25-29 \cdot 9$ & -1.95 & & -2.35 & & $-2 \cdot 28$ & \\
\hline $30-34.9$ & -3.32 & & -5.09 & & -3.44 & \\
\hline$\geq 35$ & $-8 \cdot 16$ & & $-6 \cdot 17$ & & $-7 \cdot 30$ & \\
\hline Quintile of physical activity§ & & $<0.0001$ & & $<0.0001$ & & $<0.0001$ \\
\hline 1 (low) & 0 (referent) & & 0 (referent) & & 0 (referent) & \\
\hline 2 & 1.77 & & 0.99 & & 1.04 & \\
\hline 3 & $1 \cdot 15$ & & $1 \cdot 20$ & & 0.99 & \\
\hline 4 & $2 \cdot 13$ & & 3.07 & & 3.57 & \\
\hline 5 (high) & 3.66 & & 3.79 & & 3.75 & \\
\hline Post-menopausal hormone usell & & 0.001 & & $0 \cdot 12$ & & \\
\hline 1 & 0 (referent) & & 0 (referent) & & - & \\
\hline 2 & -1.66 & & 0.17 & & - & \\
\hline 3 & $-2 \cdot 11$ & & 1.94 & & - & \\
\hline 4 & $-1 \cdot 17$ & & 1.53 & & - & \\
\hline 5 & -0.66 & & 0.71 & & - & \\
\hline Alcohol intake (g/d) & & $<0.0001$ & & $<0.001$ & & \\
\hline 0 & 0 (referent) & & 0 (referent) & & - & \\
\hline$>0$ to $<5$ & 0.24 & & 1.34 & & - & \\
\hline 5 to $<10$ & 1.33 & & $2 \cdot 38$ & & - & \\
\hline$\geq 10$ & 2.62 & & $2 \cdot 69$ & & - & \\
\hline Season of blood draw $\dagger$ & & $<0.0001$ & & $<0.0001$ & & $<0.0001$ \\
\hline Autumn & 0 (referent) & & 0 (referent) & & 0 (referent) & \\
\hline Summer & $1 \cdot 18$ & & 1.33 & & 0.88 & \\
\hline Spring & -2.68 & & -5.55 & & -3.08 & \\
\hline Winter & -3.35 & & -5.61 & & -4.45 & \\
\hline
\end{tabular}

MET, metabolic equivalents.

${ }^{*}$ Adjusted for laboratory batch.

† Age and season not used in predicted score calculation.

¥UV-B flux category (in Robertson-Berger (RB) count $\times 10^{-4}$ ): NHS: 1 is $>113,2$ is 113,3 is $<113$; NHSII: 1 is $145-196,2$ is $115-144,3$ is $108-114,4$ is $<105$; HPFS: 1 is $158-196,2$ is $137-154,3$ is $115-133,4$ is $105-113,5$ is $<105$.

§ Mean values in extreme quintiles of physical activity (in METh/week): NHS: 1 is $1.2,5$ is 41.4 ; NHSIl: 1 is $1.6,5$ is $52 \cdot 8$; HPFS: 1 is $3.6,5$ is 87.4 .

|l Post-menopausal hormone use: NHS: 1 is pre-menopausal, 2 is post-menopausal, never user, 3 is post-menopausal, past user, 4 is post-menopausal, current user, 5 is post-menopausal, unknown use; NHSII: 1 is never user, 2 is past user, 3 is recent past user, 4 is current user, 5 is unknown use. 


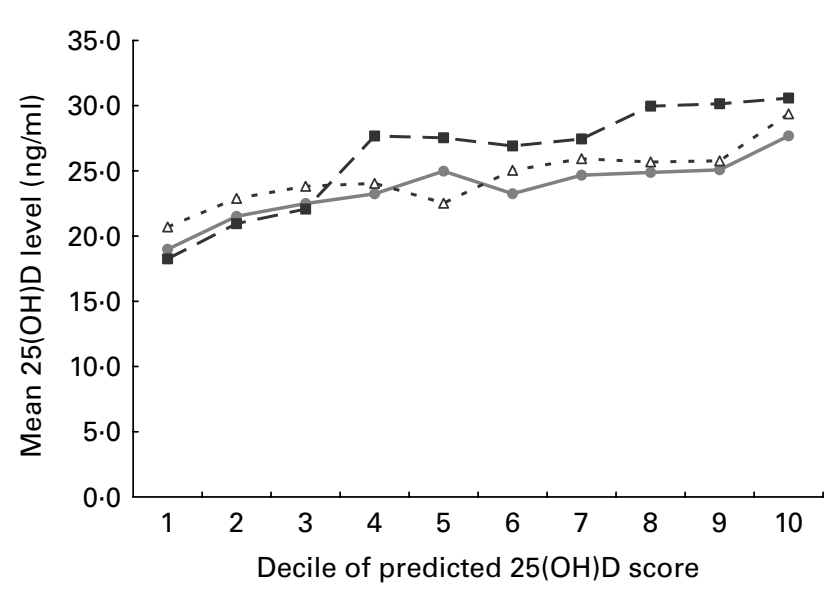

Fig. 1. Mean actual 25-hydroxyvitamin $D(25(\mathrm{OH}) \mathrm{D})$ level by decile of predicted 25(OH)D score in the Nurses' Health Studies (NHS ( $n$ 779, - - ) and NHSII $(n 445,-n-))$ and the Health Professionals Follow-up Study $(n 836,-\Delta-$ ) validation samples.

the NHS, $12.3 \mathrm{ng} / \mathrm{ml}(95 \%$ CI $8.7,16.0)$ for the NHSII and $8.7 \mathrm{ng} / \mathrm{ml}(95 \%$ CI $5.5,11.8)$ for the HPFS.

Because epidemiological studies often categorise exposures into quantiles for analysis, we cross-classified individuals in the validation samples by quintile of predicted $25(\mathrm{OH}) \mathrm{D}$ and measured plasma $25(\mathrm{OH}) \mathrm{D}$ levels to determine how well the predicted score performed in ranking individuals with respect to plasma levels. Between $24.8 \%$ (NHS) and $29.9 \%$ (NHSII) of individuals fell into identical quintiles of predicted and measured 25(OH)D. Using the predicted scores, the majority of individuals were classified in either the same quintile or the adjacent quintile of actual plasma $25(\mathrm{OH}) \mathrm{D}$ concentration (NHS: $59 \cdot 8 \%$, NHSII: $66.5 \%$, HPFS: $61.4 \%$; Fig. 2 ). Only $5 \%$ or less of participants in each cohort were in extreme opposite quintiles according to predicted and actual 25(OH)D. Among women in the lowest quintile (Q1) of actual plasma $25(\mathrm{OH}) \mathrm{D}$ in the NHS, 33\% were categorised in Q1 of the predicted score, $57 \%$ were categorised in either Q1 or Q2 and $13 \%$ were categorised in Q5. Among women in Q1 of actual plasma $25(\mathrm{OH}) \mathrm{D}$ in the NHSII, $44 \%$ were categorised in Q1 of the predicted score, 66\% were categorised in either Q1 or Q2 and 8\% were categorised in Q5. Among men in Q1 of actual plasma 25(OH)D in the HPFS, $37 \%$ were categorised in Q1 of the predicted score, $57 \%$ were categorised in either Q1 or Q2 and 10\% were categorised in Q5.

Based on data from a previously published case-control study of colorectal cancer in the NHS and $\mathrm{HPFS}^{(4)}$, the pooled multivariable $\mathrm{OR}$ for a $10 \mathrm{ng} / \mathrm{ml}$ difference in measured $25(\mathrm{OH}) \mathrm{D}$ was $0.82(95 \% \mathrm{CI} 0 \cdot 66,1 \cdot 03)$. Using the average predicted $25(\mathrm{OH}) \mathrm{D}$ score in these analyses yielded an OR of 0.78 (95\% CI $0 \cdot 41,1 \cdot 48)$.

In our reproducibility substudy in the NHS, the ICC for plasma 25(OH)D measured over 10-11 years was 0.50 (95\% CI 0.43, 0.57). Among these 443 women, the age- and season-adjusted Spearman correlation coefficient between average measured $25(\mathrm{OH}) \mathrm{D}$ based on two blood samples and long-term average predicted $25(\mathrm{OH}) \mathrm{D}$ over the same time period was $0 \cdot 23$. We corrected for within-person variation in plasma $25(\mathrm{OH}) \mathrm{D}$ to obtain a deattenuated correlation coefficient of $0 \cdot 28$.

\section{Discussion}

Using data from three US cohorts, we derived predicted $25(\mathrm{OH}) \mathrm{D}$ scores based on various factors that influence circulating levels. The determinants of circulating $25(\mathrm{OH}) \mathrm{D}$ we identified generally were consistent with the predictors reported by others ${ }^{(27-29,48-55)}$. The set of predictors included in the final models explained only a proportion of the total variability in plasma $25(\mathrm{OH}) \mathrm{D}$ levels (i.e. 25-33\%). The $r^{2}$ for our prediction models were generally consistent with previously published models ${ }^{(27-29)}$. Millen et al. ${ }^{(28)}$ reported a similar multivariable regression model with a comparable $r^{2}$ $(0 \cdot 21)$ and correlation between predicted and actual $25(\mathrm{OH}) \mathrm{D}(0.45)$ for the Women's Health Initiative. In the Framingham Offspring Study, Liu et al. ${ }^{(27)}$ developed a model to predict a $25(\mathrm{OH}) \mathrm{D}$ score based on a similar set of predictors $\left(r^{2} 0 \cdot 26\right)$, and in their validation study observed a correlation of 0.51 between predicted and actual levels. In the Adventist Health Study-2, Chan et al. ${ }^{(29)}$ reported $r^{2}$ of 0.22 and 0.33 for White and Black populations, respectively $(0.42 \mathrm{com}-$ bined); however, they did not compare predicted and actual $25(\mathrm{OH}) \mathrm{D}$ levels in an independent sample. Because only a small proportion of the total variability in plasma $25(\mathrm{OH}) \mathrm{D}$ levels is explained by identified predictors, predicted $25(\mathrm{OH}) \mathrm{D}$ scores cannot be interpreted as direct blood measurements of $25(\mathrm{OH}) \mathrm{D}$ to determine an individual's vitamin D sufficiency, insufficiency or deficiency status.

Vitamin D prediction models have potential strengths and limitations as exposure assessment tools. Our models and others' have substantial unexplained variability, which probably can be attributed to error in the measurement of predictor variables and plasma 25(OH)D levels and lack of information about other important determinants of vitamin D status such as genetic factors ${ }^{(49,56)}$ and actual UV exposure. While sun sensitivity characteristics (e.g. ability to tan, susceptibility to burn and number of lifetime sunburns) were not predictive of $25(\mathrm{OH}) \mathrm{D}$ in the NHS, data on personal sun exposure and sun behaviours (such as time spent outdoors and use of sunscreen or protective clothing), important determinants of circulating $25(\mathrm{OH}) \mathrm{D}$, were not regularly collected in these cohorts. We examined leisure-time physical activity as a proxy for time spent outdoors and found this to be a significant predictor.

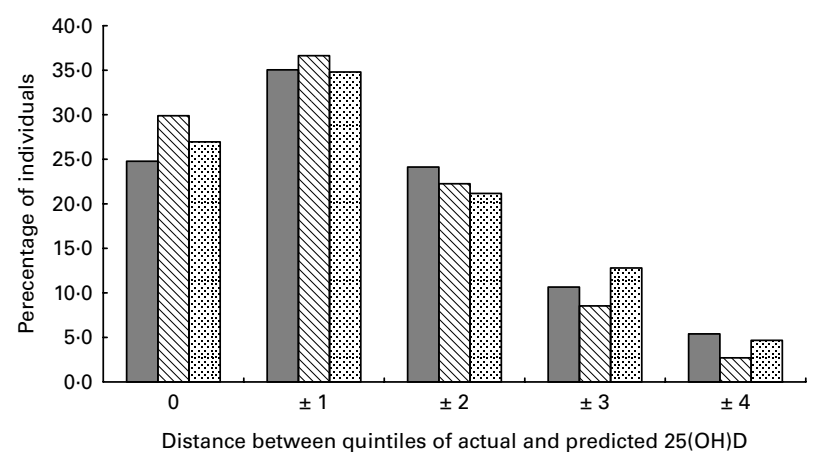

Fig. 2. Percentage of individuals classified by quintiles of actual and predicted 25-hydroxyvitamin D $(25(\mathrm{OH}) \mathrm{D})$ in the Nurses' Health Studies $(\square$, NHS and $\mathbb{\nabla}, \mathrm{NHSII})$ and the Health Professionals Follow-up Study (0) validation samples. 
The prediction models also include an estimate of average annual UV-B flux, a composite measure of mean UV-B radiation level based on latitude, altitude and cloud cover, which also was a significant determinant of circulating 25(OH)D.

Millen et al $^{(28)}$ concluded that predicted 25(OH)D scores 'do not adequately reflect serum 25(OH)D concentrations'. While we agree that predicted scores cannot substitute for blood measures in assessing current 25(OH)D level, we view the results of both studies as providing reasonable evidence that predicted $25(\mathrm{OH}) \mathrm{D}$ score is an acceptable marker of vitamin D status for the purposes of distinguishing a substantial range of vitamin $\mathrm{D}$ exposure in a given study population. In chronic disease epidemiology, the actual contrast between high and low exposure level over years or decades is particularly relevant. We calculated differences in measured $25(\mathrm{OH}) \mathrm{D}$ between extreme deciles of predicted score of 9$12 \mathrm{ng} / \mathrm{ml}$, which represents the actual contrast in long-term $25(\mathrm{OH}) \mathrm{D}$ that can be studied in these populations. This difference corresponds to differences in vitamin $\mathrm{D}$ intakes of approximately $25-37.5 \mu \mathrm{g}$ (i.e. $1000-1500 \mathrm{IU}$ )/ $\mathrm{d}^{(57)}$, and is considerably larger than what may be estimated using single surrogates of vitamin D exposure, such as dietary vitamin D intake, which explains a contrast of approximately $3 \mathrm{ng} / \mathrm{ml}$ in $25(\mathrm{OH}) \mathrm{D}$ between high and low intake (Table 1 ).

A single blood measurement of $25(\mathrm{OH}) \mathrm{D}$ has the advantage of being a direct measure of circulating 25(OH)D; however, it is substantially influenced by recent and acute exposures (e.g. beach vacation, season), which contributes to measurement error in estimating long-term 25(OH)D. Correlations between two direct $25(\mathrm{OH}) \mathrm{D}$ measures taken $2-14$ years apart range from 0.42 to $0.72^{(17-20)}$, reflecting that a single $25(\mathrm{OH}) \mathrm{D}$ measurement is not a true 'gold standard' of long-term $25(\mathrm{OH}) \mathrm{D}$ level. In the NHS, the ICC for plasma $25(\mathrm{OH}) \mathrm{D}$ measured $2-3$ years apart was $0 \cdot 72^{(17)}$; over $10-11$ years, the ICC was 0.50 . While an ICC of 0.50 indicates fair to good reproducibility of a biomarker ${ }^{(58)}$, the difference between the 2-3-year ICC and the 10-11-year ICC reflects lower reproducibility over a longer time period. Therefore, in our analyses and those by Millen et $a l^{(28)}$ and Liu et $a l^{(27)}$, correlation coefficients comparing predicted and actual $25(\mathrm{OH}) \mathrm{D}$ are probably underestimated because measured $25(\mathrm{OH}) \mathrm{D}$ is not a true 'gold standard' and because random within-person error in the measurement of both variables attenuates correlation coefficients ${ }^{(32)}$. Because circulating plasma $25(\mathrm{OH}) \mathrm{D}$ is an imperfect measure of long-term $25(\mathrm{OH}) \mathrm{D}$ status, the comparison of mean actual $25(\mathrm{OH}) \mathrm{D}$ level by category of predicted score in validation analyses may better reflect the utility of predicted $25(\mathrm{OH}) \mathrm{D}$ scores to assess long-term 25(OH)D status. Although we assumed that the average of two plasma $25(\mathrm{OH}) \mathrm{D}$ measurements taken 10-11 years apart would better represent long-term $25(\mathrm{OH}) \mathrm{D}$ status, correlation coefficients were similar in the NHS sample with repeated measurements.

Another objection commonly raised about the predicted $25(\mathrm{OH}) \mathrm{D}$ score is that it may be confounded by its predictors (e.g. physical activity or BMI), which could be independent risk factors for disease ${ }^{(28)}$. This criticism would also be true for plasma $25(\mathrm{OH}) \mathrm{D}$ levels, which inherently incorporate these factors. Importantly, including predictors of vitamin D status as covariates in multivariable models may represent over-adjustment because these variables are important determinants of $25(\mathrm{OH}) \mathrm{D}$. Therefore, adjusting for these factors may be inappropriate. A potential advantage of using predicted 25(OH)D scores over measured 25(OH)D in analytical epidemiology is that a sensitivity analysis could be performed in which physical activity (or other predictor) is excluded from the score, thereby removing potential confounding by this factor. In practice, however, we did not observe evidence of confounding of predicted $25(\mathrm{OH}) \mathrm{D}$ by BMI or physical activity in previous analyses of colorectal cancer risk in the HPFS ${ }^{(18)}$.

Predicted scores were derived based on data not collected for assessing vitamin D status; the predictive ability of derived scores would probably improve if additional determinants of circulating $25(\mathrm{OH}) \mathrm{D}$, such as personal sun exposure behaviours, were incorporated. Random measurement error in predicted $25(\mathrm{OH}) \mathrm{D}$ is expected to attenuate measures of association with disease ${ }^{(32,59)}$; however, predicted scores should allow investigators to test a sizeable contrast in 25(OH)D between 'low' and 'high' exposure categories and will still be useful to detect moderate to strong vitamin D-disease associations. In our cohorts, we observe similar associations for various disease endpoints using plasma $25(\mathrm{OH}) \mathrm{D}$ and predicted $25(\mathrm{OH}) \mathrm{D}$ as the exposure variable, including hypertension $^{(8)}$, colorectal cancer incidence ${ }^{(18)}$ and survi$\mathrm{val}^{(34,60)}$, pancreatic cancer incidence ${ }^{(33)}$ (E Giovannucci, unpublished results) and prostate cancer incidence ${ }^{(18)}$ (E Giovannucci, unpublished results). For example, although statistical power was reduced when we used average predicted $25(\mathrm{OH}) \mathrm{D}$, we observed similar OR of approximately 0.8 for a $10 \mathrm{ng} / \mathrm{ml}$ difference in either plasma or predicted $25(\mathrm{OH}) \mathrm{D}$ for colorectal cancer based on data from a previous case-control study in the NHS and HPFS ${ }^{(4)}$. In a much larger HPFS cohort analysis with 691 colorectal cancer cases, the relative risk for the same increment of predicted $25(\mathrm{OH}) \mathrm{D}$ was $0.63(95 \% \mathrm{CI} 0.48,0.83)^{(18)}$, demonstrating that the loss in precision may be recovered by increasing the sample size in analyses using predicted scores.

For analyses of vitamin $\mathrm{D}$ and chronic diseases in these specific cohorts, predicted $25(\mathrm{OH}) \mathrm{D}$ scores can be derived for each participant at each questionnaire cycle. An advantage of longitudinal data is the availability of updated predictor information, allowing the predicted $25(\mathrm{OH}) \mathrm{D}$ score to change over time and potentially better capturing long-term average vitamin D status. Such studies would use data available from the full cohorts and complement biomarker analyses with smaller sample sizes. As noted by others ${ }^{(29)}$, prediction models developed in the NHS, NHSII and HPFS may not apply to other study populations because of underlying population differences and/or availability of data; however, similar models may be developed using the general approach described here and could be useful for investigating hypothesised associations between vitamin $\mathrm{D}$ status and disease. It is also possible, however, that the prediction models developed in these cohorts could perform well in populations with similar demographics (e.g. male and female populations with similar age, race and residential latitude distributions as 
the NHS, NHSII and HPFS); such applications would benefit from additional validation. In conclusion, predicted $25(\mathrm{OH}) \mathrm{D}$ scores may be a practical alternative for studying such associations in international and other settings where large-scale biomarker studies are not feasible.

\section{Acknowledgements}

The present study was supported by the National Institutes of Health (CA87969, CA49449, CA050385, CA67262 and CA55075). K. A. B. was supported by the Training Program in Environmental Health Sciences (T32 ES007155) and the Nutritional Epidemiology of Cancer Education and Career Development Program (R25 CA098566). E. G. secured funding, and with K. A. B., D. F., M. D. H. and F. L., conceived and designed this study. D. F. and E. G. oversaw the study's implementation and analytic strategy. K. A. B., A. H. E., K. W., D. F. and E. G. were involved in data collection while K. A. B., D. F., Y. L. and S. M. conducted the data analyses, with additional statistical support from A. H. E. and K. W. All authors contributed to interpretation of the results. K. A. B. wrote the first draft of the manuscript, which was critically revised and approved by all authors. The authors assert that they have no conflicts of interest. Finally, the authors thank Dr Walter Willett for his scientific input on this paper.

\section{References}

1. Garland CF, Gorham ED, Mohr SB, et al. (2007) Vitamin D and prevention of breast cancer: pooled analysis. J Steroid Biochem Mol Biol 103, 708-711.

2. Giovannucci E (2005) The epidemiology of vitamin D and cancer incidence and mortality: a review (United States). Cancer Causes Control 16, 83-95.

3. Li H, Stampfer MJ, Hollis JB, et al. (2007) A prospective study of plasma vitamin D metabolites, vitamin D receptor polymorphisms, and prostate cancer. PLoS Med 4, e103.

4. Wu K, Feskanich D, Fuchs CS, et al. (2007) A nested case control study of plasma 25-hydroxyvitamin D concentrations and risk of colorectal cancer. J Natl Cancer Inst 99, $1120-1129$.

5. IARC (2008) Vitamin D and Cancer. IARC Working Group Reports Vol. 5. In IARC Working Group Reports Vol. 5. Lyon: International Agency for Research on Cancer.

6. Holick MF (2004) Sunlight and vitamin D for bone health and prevention of autoimmune diseases, cancers, and cardiovascular disease. Am J Clin Nutr 80, 1678S-1688S.

7. Giovannucci E, Liu Y, Hollis BW, et al. (2008) 25-Hydroxyvitamin D and risk of myocardial infarction in men: a prospective study. Arch Intern Med 168, 1174-1180.

8. Forman JP, Giovannucci E, Holmes MD, et al. (2007) Plasma 25-hydroxyvitamin D levels and risk of incident hypertension. Hypertension 49, 1063-1069.

9. Michos ED \& Melamed ML (2008) Vitamin D and cardiovascular disease risk. Curr Opin Clin Nutr Metab Care 11, 7-12.

10. Pittas AG, Lau J, Hu FB, et al. (2007) The role of vitamin D and calcium in type 2 diabetes. A systematic review and meta-analysis. J Clin Endocrinol Metab 92, 2017-2029.

11. Pittas AG, Chung M, Trikalinos T, et al. (2010) Systematic review: vitamin $\mathrm{D}$ and cardiometabolic outcomes. Ann Intern Med 152, 307-314.
12. Feskanich D, Willett WC \& Colditz GA (2003) Calcium, vitamin D, milk consumption, and hip fractures: a prospective study among postmenopausal women. Am J Clin Nutr 77, 504-511.

13. Bischoff-Ferrari HA, Willett WC, Wong JB, et al. (2005) Fracture prevention with vitamin D supplementation: a meta-analysis of randomized controlled trials. JAMA 293, 2257-2264.

14. Cauley JA, Lacroix AZ, Wu L, et al. (2008) Serum 25-hydroxyvitamin $\mathrm{D}$ concentrations and risk for hip fractures. Ann Intern Med 149, 242-250.

15. Holick MF (2007) Vitamin D deficiency. N Engl J Med 357, $266-281$

16. Horst R, Reinhardt $T$ \& Reddy G (2005) Vitamin D metabolism. In Vitamin D, pp. 15-36 [D Feldman, J Pike and F Glorieux, editors]. Burlington, MA: Elsevier Academic Press.

17. Kotsopoulos J, Tworoger SS, Campos H, et al. (2010) Reproducibility of plasma and urine biomarkers among premenopausal and postmenopausal women from the Nurses' Health Studies. Cancer Epidemiol Biomarkers Prev 19, 938-946.

18. Giovannucci E, Liu Y, Rimm EB, et al. (2006) Prospective study of predictors of vitamin D status and cancer incidence and mortality in men. J Natl Cancer Inst 98, 451-459.

19. Hofmann JN, Yu K, Horst RL, et al. (2010) Long-term variation in serum 25-hydroxyvitamin D concentration among participants in the Prostate, Lung, Colorectal, and Ovarian Cancer Screening Trial. Cancer Epidemiol Biomarkers Prev 19, 927-931.

20. Jorde R, Sneve M, Hutchinson M, et al. (2010) Tracking of serum 25-hydroxyvitamin D levels during 14 years in a population-based study and during 12 months in an intervention study. Am J Epidemiol 171, 903-908.

21. Garland CF \& Garland FC (1980) Do sunlight and vitamin D reduce the likelihood of colon cancer? Int J Epidemiol 9 , 227-231.

22. Garland FC, Garland CF, Gorham ED, et al. (1990) Geographic variation in breast cancer mortality in the United States: a hypothesis involving exposure to solar radiation. Prev Med 19, 614-622.

23. Grant WB \& Mohr SB (2009) Ecological studies of ultraviolet B, vitamin D and cancer since 2000. Ann Epidemiol 19 446-454.

24. Anderson LN, Cotterchio M, Vieth R, et al. (2010) Vitamin D and calcium intakes and breast cancer risk in pre- and postmenopausal women. Am J Clin Nutr 91, 1699-1707.

25. Martinez ME, Giovannucci EL, Colditz GA, et al. (1996) Calcium, vitamin D, and the occurrence of colorectal cancer among women. J Natl Cancer Inst 88, 1375-1382.

26. Oh K, Willett WC, Wu K, et al. (2007) Calcium and vitamin D intakes in relation to risk of distal colorectal adenoma in women. Am J Epidemiol 165, 1178-1186.

27. Liu E, Meigs JB, Pittas AG, et al. (2010) Predicted 25-hydroxyvitamin $\mathrm{D}$ score and incident type 2 diabetes in the Framingham Offspring Study. Am J Clin Nutr 91, 1627-1633.

28. Millen AE, Wactawski-Wende J, Pettinger M, et al. (2010) Predictors of serum 25-hydroxyvitamin D concentrations among postmenopausal women: the Women's Health Initiative Calcium plus Vitamin D clinical trial. Am J Clin Nutr 91, $1324-1335$.

29. Chan J, Jaceldo-Siegl K \& Fraser GE (2010) Determinants of serum 25 hydroxyvitamin D levels in a nationwide cohort of blacks and non-Hispanic whites. Cancer Causes Control 21 501-511. 
30. Wacholder S, Armstrong B \& Hartge P (1993) Validation studies using an alloyed gold standard. Am J Epidemiol 137, 1251-1258.

31. Spiegelman D, Schneeweiss S \& McDermott A (1997) Measurement error correction for logistic regression models with an "alloyed gold standard". Am J Epidemiol 145, 184-196.

32. Willett W (1998) Nutritional Epidemiology, 2nd ed. New York, NY: Oxford University Press.

33. Bao Y, Ng K, Wolpin BM, et al. (2010) Predicted vitamin D status and pancreatic cancer risk in two prospective cohort studies. Br J Cancer 102, 1422-1427.

34. Ng K, Wolpin BM, Meyerhardt JA, et al. (2009) Prospective study of predictors of vitamin D status and survival in patients with colorectal cancer. Br J Cancer 101, 916-923.

35. Willett WC, Sampson L, Stampfer MJ, et al. (1985) Reproducibility and validity of a semiquantitative food frequency questionnaire. Am J Epidemiol 122, 51-65.

36. Willett WC, Sampson L, Browne ML, et al. (1988) The use of a self-administered questionnaire to assess diet four years in the past. Am J Epidemiol 127, 188-199.

37. Rimm EB, Giovannucci EL, Stampfer MJ, et al. (1992) Reproducibility and validity of an expanded self-administered semiquantitative food frequency questionnaire among male health professionals. Am J Epidemiol 135, 1114-1126, discussion 1127-1136.

38. Feskanich D, Rimm EB, Giovannucci EL, et al. (1993) Reproducibility and validity of food intake measurements from a semiquantitative food frequency questionnaire. $J \mathrm{Am}$ Diet Assoc 93, 790-796.

39. Hollis BW (1997) Quantitation of 25-hydroxyvitamin D and 1,25-dihydroxyvitamin $\mathrm{D}$ by radioimmunoassay using radioiodinated tracers. Methods Enzymol 282, 174-186.

40. Gallicchio L, Helzlsouer KJ, Chow WH, et al. (2010) Circulating 25-hydroxyvitamin $\mathrm{D}$ and the risk of rarer cancers: design and methods of the cohort consortium vitamin D pooling project of rarer cancers. Am J Epidemiol 172, 10-20.

41. Wagner D, Hanwell HE \& Vieth R (2009) An evaluation of automated methods for measurement of serum 25-hydroxyvitamin D. Clin Biochem 42, 1549-1556.

42. Hollis BW (2008) Measuring 25-hydroxyvitamin D in a clinical environment: challenges and needs. Am J Clin Nutr 88, 507S-510S.

43. Willett WC, Howe GR \& Kushi LH (1997) Adjustment for total energy intake in epidemiologic studies. Am J Clin Nutr $\mathbf{6 5}$, 1220S-1228S, discussion 1229S-1231S.

44. Scotto J, Fears TR \& Fraumeni JF Jr (1996) Solar radiation. In Cancer Epidemiology and Prevention, pp. 355-372 [D Schottenfeld and JF Fraumeni Jr, editors]. New York, NY: Oxford University Press.

45. DerSimonian R \& Laird N (1986) Meta-analysis in clinical trials. Control Clin Trials 7, 177-188.
46. Beaton GH, Milner J, Corey P, et al. (1979) Sources of variance in 24-hour dietary recall data: implications for nutrition study design and interpretation. Am J Clin Nutr 32, $2546-2559$.

47. Liu K, Stamler J, Dyer A, et al. (1978) Statistical methods to assess and minimize the role of intra-individual variability in obscuring the relationship between dietary lipids and serum cholesterol. J Chronic Dis 31, 399-418.

48. McCullough ML, Weinstein SJ, Freedman DM, et al. (2010) Correlates of circulating 25-hydroxyvitamin D: Cohort Consortium Vitamin D Pooling Project of Rarer Cancers. Am J Epidemiol 172, 21-35.

49. Shea MK, Benjamin EJ, Dupuis J, et al. (2009) Genetic and non-genetic correlates of vitamins K and D. Eur J Clin Nutr 63, 458-464.

50. Kelly JL, Friedberg JW, Calvi LM, et al. (2010) A case-control study of ultraviolet radiation exposure, vitamin $\mathrm{D}$, and lymphoma risk in adults. Cancer Causes Control 21, 1265-1275.

51. Benjamin A, Moriakova A, Akhter N, et al. (2009) Determinants of 25-hydroxyvitamin D levels in African-American and Caucasian male veterans. Osteoporos Int 20, 1795-1803.

52. Holick MF, Siris ES, Binkley N, et al. (2005) Prevalence of vitamin $\mathrm{D}$ inadequacy among postmenopausal North American women receiving osteoporosis therapy. J Clin Endocrinol Metab 90, 3215-3224.

53. Burgaz A, Akesson A, Oster A, et al. (2007) Associations of diet, supplement use, and ultraviolet $\mathrm{B}$ radiation exposure with vitamin D status in Swedish women during winter. Am J Clin Nutr 86, 1399-1404.

54. Jacques PF, Felson DT, Tucker KL, et al. (1997) Plasma 25-hydroxyvitamin $\mathrm{D}$ and its determinants in an elderly population sample. Am J Clin Nutr 66, 929-936.

55. Brock K, Huang WY, Fraser DR, et al. (2010) Low vitamin D status is associated with physical inactivity, obesity and low vitamin D intake in a large US sample of healthy middleaged men and women. J Steroid Biochem Mol Biol 121, 462-466.

56. Engelman CD, Fingerlin TE, Langefeld CD, et al. (2008) Genetic and environmental determinants of 25-hydroxyvitamin D and 1,25-dihydroxyvitamin D levels in Hispanic and African Americans. J Clin Endocrinol Metab 93, 3381-3388.

57. Heaney RP, Davies KM, Chen TC, et al. (2003) Human serum 25-hydroxycholecalciferol response to extended oral dosing with cholecalciferol. Am J Clin Nutr 77, 204-210.

58. Rosner B (2006) Fundamentals of Biostatistics. Belmont, CA: Duxbury.

59. Thomas D, Stram D \& Dwyer J (1993) Exposure measurement error: influence on exposure-disease. Relationships and methods of correction. Annu Rev Public Health 14, 69-93.

60. Ng K, Meyerhardt JA, Wu K, et al. (2008) Circulating 25-hydroxyvitamin D levels and survival in patients with colorectal cancer. J Clin Oncol 26, 2984-2991. 